\title{
Dispersão de Lernaea cyprinacea (Linnaeus) (Crustacea, Copepoda) na região norte do estado do Paraná, Brasil
}

\author{
Mário Artur Gabrielli ${ }^{1}$ \\ Mário Luís Orsi ${ }^{1}$
}

\begin{abstract}
Dispersion of Lernaea cyprinacea (Linnaeus) (Crustacea, Copepoda) in the north area of the Paraná State, Brasil. Inside of the parasitoses context in fish, the study was successful to us verified the lerniose was present in the north area of the State. It had reached the native fish in the basin of the river Tibagi. For the study, visits were made to the aquaculture and samples collected in the area of Sertanopolis on the river Tibagi. We verified that the lerniose was growing in the area and had already reached several species of native fish of the river Tibagi. This concern for all the researchers of the area, as well as the producers of fish.

KEY WORDS. Lernaea cyprinacea, Tibagi, Paranapanema, river
\end{abstract}

Os Copepoda são os mais numerosos dos crustáceos parasitas e sendo talvez, o grupo mais comum de parasitos de peixes. Os copépodes lerneídeos são encontrados parasitando tegumento, brânquias, olhos, nadadeiras e até mesmo dentro do aparelho bucal, junto ao palato e narinas dos peixes. Permanecem aderidos a estas regiões por órgãos de fixação, resultantes das adaptações morfológicas da região cefálica destes parasitos (EIRAS 1994).

Uma grande revisão foi realizada por KABATA $(1970,1979,1981)$, sobre a interação desses crustáceos parasitos e seus hospedeiros. Os principais danos causados pelo parasito nos peixes, parecem ser nas brânquias, provocando hemorragias e necroses, com conseqüente diminuição da eficiência respiratória. Algumas espécies de lerneídeos causam também, lesões profundas no tegumento, na musculatura externa, na superfície dos olhos e em alguns casos, em órgãos internos. $\mathrm{Na}$ maioria das vezes, os danos causados por estes parasitos, aos peixes hospedeiros, possibilitam infecções secundárias, com a penetração de outros agentes patogênicos como bactérias, fungos e vírus.

Diversos autores demonstraram ainda, que infestações com copépodes em peixes, podem levar a perda de peso, redução da taxa de crescimento, alterações no quadro sangüíneo e mudanças no comportamento como, natação errática, letargia e fricção do corpo contra superfícies ásperas (OSTRENSKY \& BOEGER 1998).

Dos copépodes conhecidos no Brasil, a espécie mais estudada nos últimos anos é sem dúvida, Lernaea cyprinacea (Linnaeus, 1758), introduzida há alguns anos, com o início do cultivo da carpa húngara Cyprinus carpio Linnaeus, 1758, como espécie hospedeira. Atualmente, o parasito ocorre em quase todos os estados brasileiros, sendo registrado principalmente em propriedades agrícolas com ativi-

1) Departamento de Biologia Animal e Vegetal, Centro de Ciências Biológicas, Universidade

Estadual de Londrina. Caixa Postal 6001, 86051-990 Londrina, Paraná, Brasil. 
dade de piscicultura (BOEGER \& SANTOS-NETO 1993), onde enormes prejuízos vem sendo causados por este parasito, aos produtores de peixes. $O$ tratamento dos peixes é de difícil execução, sendo necessário o emprego de produtos altamente tóxicos (PAVANElli et al. 1998; OSTRENSKy \& BOEgER 1998) e, por vezes, medidas mais drásticas como por exemplo a eliminação de todo o plantel de peixes.

Apesar desses parasitas terem sido introduzidos, parasitando carpas, existe a possibilidade de já estarem parasitando peixes nativos nos rios brasileiros. Esta hipótese tem fundamento nos escapes massivos de peixes das pisciculturas para os ambientes naturais, o qual pode viabilizar a disseminação de patógenos e parasitos, para os rios, como já observado por ORSI \& AGOSTINHO (1999).

Em vista destas informações a respeito destes lerneídeos, fez-se um estudo sobre a ocorrência do parasitismo, tanto em algumas propriedades com atividade de piscicultura, situadas em municípios que compõem parte das bacias do Rio Tibagi, Rio das Cinzas e Rio Paranapanema, como também, observações no próprio Rio Tibagi (baixo Rio Tibagi) (Fig. 1). Todas as áreas de estudo são pertencentes à região norte do estado do Paraná. Foram incluídas nesta análise, tanto as espécies de peixes de interesse comercial (piscicultura) como algumas espécies nativas da região, parasitadas por lerneídeos.

Aproveitando-se do convênio entre Universidade Estadual de Londrina e a Cooperativa Agrícola de Rolândia (COROL), para prestação de assistência técnica aos piscicultores, dados sobre a infestação de lerneídeos em peixes de pisciculturas foram obtidos. Para realização do trabalho foram estabelecidas de forma fixa, visitas em 53 propriedades pertencentes a 15 municípios, no período de 1995 a 1998, sendo entrevistados os proprietários, e ou, responsáveis, indagando-se sobre as condições das espécies criadas, bem como a existência de problemas sanitários nos peixes, como parasitoses, mortalidade e ausência de crescimento no plantel.

Posteriormente à entrevista, procedeu-se a uma vistoria nas instalações de cada piscicultura, obtendo-se uma noção básica do ambiente dos viveiros. Os dados dessas entrevistas e observações foram acompanhados por uma amostra de peixes de cada espécie criada (mínimo de cinco indivíduos), coletados em cada uma das propriedades e assim analisados com lupa manual, quanto à infestação por lerneídeos e conseqüente identificação destes. Os dados foram agrupados em freqüência absoluta de propriedades por município, onde casos de lerniose foram registrados, conforme descrito na tabela I. Para as espécies de peixes criados em cativeiro com registro de lerniose, os dados foram transformados em freqüência relativa (\%), prevalência do parasita e os meses de maior incidência de infestação foram anotados, para o total de propriedades analisadas (Tab. II).

Nas observações sobre espécies nativas em ambiente natural, foram realizados dois períodos de coleta de peixes, sendo de 1994-1995 e 1997-1998. Todas as coletas foram realizadas na região do baixo Rio Tibagi, trecho de Sertanópolis, Paraná. Os peixes coletados foram identificados e examinados em lupa manual, para verificação da ocorrência do ectoparasita. Em caso positivo os parasitos foram retirados das estruturas dos peixes, onde estavam aderidos e colocados em frascos contendo a substância AFA. Sendo então levados para o laboratório de ictiologia da Universidade Estadual de Londrina. 


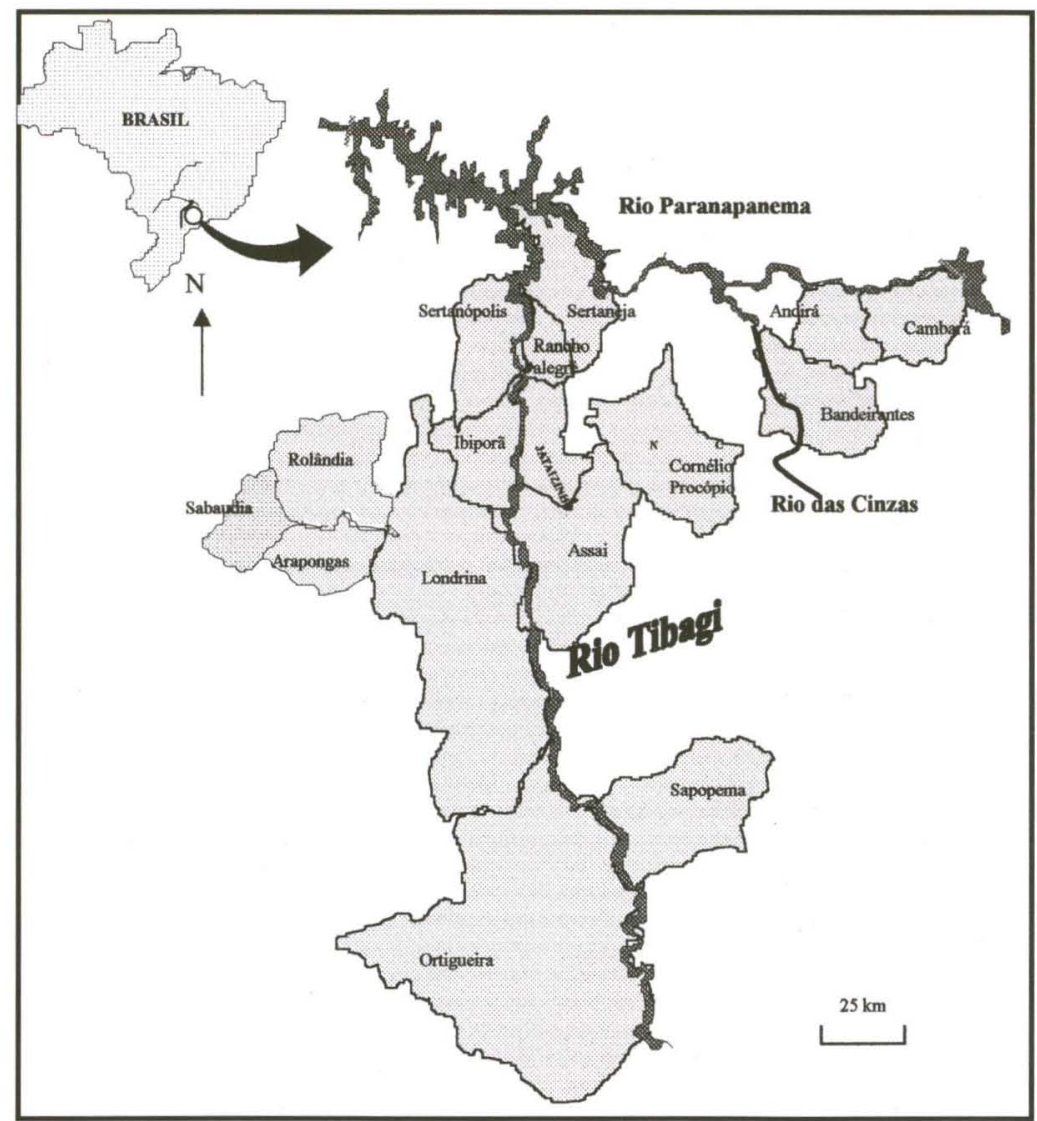

Fig. 1. Mapa da área de estudo.

Tabela I. Freqüência absoluta de propriedades onde ocorreram casos de Lerniose por Lernaea cyprinacea no período de 1995 até 1998, nos municipios da região norte do Paraná.

\begin{tabular}{lcccc}
\hline & \multicolumn{4}{c}{ Número de propriedades com casos de Lerniose } \\
\cline { 2 - 5 } \multicolumn{1}{c}{ Municípió } & 1995 & 1996 & 1997 & 1998 \\
\hline Cambará & 1 & 2 & 3 & 2 \\
Andirá & 0 & 2 & 2 & 6 \\
Itambaracá & 0 & 1 & 3 & 5 \\
Bandeirantes & 0 & 2 & 3 & 3 \\
Cornélio Procópio & 1 & 1 & 2 & 1 \\
Uraí & 0 & 0 & 2 & 5 \\
Rancho Alegre & 2 & 0 & 4 & 3 \\
Assaí & 0 & 0 & 2 & 1 \\
Londrina & 1 & 0 & 1 & 2 \\
Cambé & 0 & 1 & 2 & 1 \\
Rolándia & 1 & 1 & 5 & 3 \\
Sapopema & 0 & 0 & 2 & 2 \\
Ortigueira & 0 & 1 & 3 & 1 \\
Ibiporă & 0 & 1 & 2 & 3 \\
Sabáudia & 0 & 0 & 2 & 42 \\
\hline Total & 6 & 12 & 36 & 3 \\
\hline
\end{tabular}


Tabela II. Prevalência do parasito em espécies de peixes criadas comercialmente, sendo atingidas pela Lerniose e os meses de maior incidência do Parasita, no período de 1995 a 1998, nas propriedades analisadas da região norte do Paraná.

\begin{tabular}{|c|c|c|c|c|c|c|c|c|}
\hline \multirow[t]{2}{*}{ Espécies } & \multirow{2}{*}{$\begin{array}{c}\text { Número de } \\
\text { peixes analisados }\end{array}$} & \multirow{2}{*}{$\begin{array}{l}\text { Prevalência da } \\
\text { infestação (\%) }\end{array}$} & \multicolumn{6}{|c|}{ Meses de maior incidència } \\
\hline & & & J & $\mathrm{FMAN}$ & $1 \mathrm{~J} \mathrm{~J} \mathrm{~A}$ & A S & 0 & N D \\
\hline Piaractus mesopotamicus Holmberg, 1886 & 203 & 95 & $\mathrm{X}$ & $\mathrm{x}$ & & & & $x \times$ \\
\hline Cyprinus carpio Linneaus, 1758 & 169 & 100 & $\mathrm{X}$ & $x \times$ & & &  & $x \times$ \\
\hline Leporinus macrocephalus Garavello \& Britski, 1987 & 194 & 100 & $\mathrm{x}$ & $\times \times \times$ & & & $(x)$ & $x \times$ \\
\hline Prochilodus lineatus Valenciennes, 1847 & 127 & 70 & $\mathrm{x}$ & $x$ & & & & \\
\hline Hibrido - Tambacu Cuvier, 1818 & 68 & 92 & $\mathrm{x}$ & $\mathrm{x} \times$ & & $\mathrm{x}$ & $x$ & $x \times$ \\
\hline Clarias gariepinus Burchell, 1822 & 112 & 10 & & $x$ & & & & \\
\hline Oreochromis niloticus Linnaeus, 1758 & 237 & 1 & $\mathrm{x}$ & & & & & \\
\hline Brycon cephalus Gunther, 1869 & 38 & 100 & $\mathrm{x}$ & $\times \times \times$ & $x \times$ & $\mathrm{x}$ & $x$ & $x \times$ \\
\hline Pseudoplatystoma corruscans Humboldt, 1833 & 29 & 2 & $\mathrm{x}$ & & & & & \\
\hline
\end{tabular}

Os parasitos após esse procedimento, foram identificados e conservados em álcool $70 \%$ glicerinado, ou preparados em lâminas permanentes, segundo o método de práticas de parasitologia de AMATO et al. (1991). Todo o material coletado está depositado na Coleção Zoológica da Universidade Estadual de Londrina.

$\mathrm{O}$ parasito considerado neste estudo como o causador da lerniose nos peixes foi Lernaea cyprinacea, que possui forma alongada, região cefálica bastante modificada em forma de chifres "anchor worm", medindo de 3,7 $\mathrm{mm}$ a $13,4 \mathrm{~mm}$ de comprimento. Foram encontradas somente fêmeas grávidas parasitando os peixes, portando cada uma, dois sacos ovígeros com cerca de 70 ovos cada um.

Um dos fatos mais importantes constatado nesse estudo, é o aumento do número de casos de lerniose no decorrer do período analisado, principalmente no ano de 1998 (42 casos), podendo destacar os municípios de Andirá, Bandeirantes, Itambaracá e Rancho Alegre, com maior número de propriedades atingidas pelo parasito, bem como a incidência do mesmo em quase todo o período de análise (Tab. I).

Nas espécies em piscicultura, observa-se que quatro espécies tiveram cerca de $100 \%$ de incidência parasitária: Cyprinus carpio Linnaeus, 1758, Leporinus macrocephalus Garavello \& Britski, 1987, Brycon cephalus Gunther, 1829, e Piaractus mesopotamicus Holmberg, 1886.

Os valores mais elevados da incidência parasitária, ocorreram nos meses em que as temperaturas atingiram valores mais altos, ou seja, de setembro a março.

Para a análise da lerniose dos peixes no Rio Tibagi, devido à metodologia de coleta empregada, poucos peixes foram capturados (Tab. III) porém, o número de espécies com infestação do parasito, aumentou de duas espécies (Leporinus elongatus Valenciennes, 1849 e Serrasalmus spilopleura Kner, 1860) no primeiro período (94-95), para sete espécies de peixes no segundo período de captura (97-98).

A lerniose tem sido um dos fatores de maior preocupação tanto para os piscicultores, como para os ictioparasitologistas. No caso da infestação por Lernaea cyprinacea em propriedades do norte do Paraná, conforme observado no presente estudo, pode ser devido aos seguintes fatores: a) lotes de peixes contaminados adquiridos pelos produtores e conseqüente ausência de quarentena nas propriedades; b) transporte inadequado dos peixes entre propriedades sem nenhum controle sanitário; c) contaminação da água de abastecimento, que não possui monitoramento permanente; e) facilidade de adaptação do parasito as condições ambientais vigentes na região. 
Não obstante o alerta dos profissionais da área de sanidade animal e técnicos de apoio, sobre os procedimentos de prevenção contra parasitoses, as infestações parasitárias vêm aumentando ano a ano em todos os municípios analisados, como no caso da lerniose. Acreditamos que tal fato, deva-se à negligência dos produtores, bem como dos órgãos responsáveis pelo assunto.

Já quanto à infestação das espécies nativas em ambientes naturais, a complexidade do problema é maior, visto que a lerniose pode dispersar-se sem controle, o que pede o envolvimento urgente de órgãos federais e estaduais relacionados ao meio ambiente, saúde e outras instituições, que possam discutir e regulamentar normas que evitem e previnam a dispersão da lerniose e outras parasitoses, que podem se originar das pisciculturas.

Tabela III. Intensidade parasitária em espécies de peixes nativos com lerniose por Lernaea cyprinacea coletadas na bacia do Rio Tibagi, nos períodos de 1994 a 1995 e 1997 a 1998. (P) Parasitado; (NP) não parasitado.

\begin{tabular}{|c|c|c|c|c|c|c|}
\hline \multirow{3}{*}{ Espécies } & \multicolumn{4}{|c|}{ Peixes coletados no período } & \multicolumn{2}{|c|}{ Intensidade parasitária média } \\
\hline & \multicolumn{2}{|c|}{$94-95$} & \multicolumn{2}{|c|}{$97-98$} & \multirow[t]{2}{*}{$94-95$} & \multirow[t]{2}{*}{$97-98$} \\
\hline & $P$ & NP & $P$ & NP & & \\
\hline Astyanax bimaculatus Linnaeus, 1758 & 0 & 106 & 9 & 163 & 0,0 & 8,0 \\
\hline Iheringichtys labrosus Kroeyer, 1874 & 0 & 39 & 1 & 21 & 0,0 & 3,0 \\
\hline Leporinus elongatus Valenciennes, 1849 & 1 & 23 & 2 & 9 & 3,0 & 5,0 \\
\hline Leporinus friderici Bloch, 1794 & 0 & 24 & 2 & 11 & 0,0 & 2,0 \\
\hline Schizodon intermedius Garavello \& Britski, 1990 & 0 & 103 & 5 & 18 & 0,0 & 1,6 \\
\hline Serrasalmus spilopleura Kner, 1860 & 2 & 34 & 6 & 15 & 2,0 & 2,5 \\
\hline Schizodon nasutus Kner, 1859 & 0 & 19 & 1 & 8 & 0,0 & 2,0 \\
\hline
\end{tabular}

AGRADECIMENTOS. Agradecemos o apoio da COROL em nome de Wellington Garcia e Ailton Demito e ao Técnico Edson Santana da Silva (UEL/BAV).

\section{REFERÊNCIAS BIBLIOGRÁFICAS}

Amato, J.F.R.; W.A. Boeger \& S.B. Amato. 1991. Coleta e Processamento de Parasitos do Pescado

- Protocolo para laboratório. Rio de Janeiro, Ed. UFFRJ, 81 p.

Boeger, W.A. \& Santos-Nfto. 1993. Lernaea, uma dor de cabeça para os piscicultores. Panorama Aquicultura, Rio de Janeiro, (3): 17.

EiRAS, J.C. 1994. Elementos da Ictioparasitologia. Porto, Fundação Eng. Antônio de Almeida, 339p. KabatA, Z. 1970. Crustacea as Enemies of Fishes. New York, THF Publ., 171p.

1979. Parasitic Copepoda of British Fishes. London, Ray Society, 468p.

1981. Copepoda (Crustacea) parasitic on fïshes: problems and perpectives. Adv. Parasitol., New York, 19: 1-71.

ORSI, M.L.\& A.A. AGOSTINHO. 1999. Introdução de espécies de peixes por escapes acidentais de tanques de cultivo em Rios da Bacia do Rio Paraná, Brasil. Revta. bras. Zool. 16 (2): 557-560.

Ostrensky, A. \& W.A. Boeger. 1998. Piscicultura Fundamentos e Técnicas de Manejo. Guaíba, Agropecuária (Ed.), $211 \mathrm{p}$.

Pavanelli, G.C.; J.C. Eiras; R.M. Takemoto. 1998. Doenças de Peixes - Profilaxia, Diagnóstico e

Tratamento. Maringá, Nupelia, Ed. Univ. Estadual Maringá, 264p. 